\title{
Future of Medical Research in Rare Diseases and Cancers: Shift from Pharma to Biotech and the Golden Age of Medical Advancement
}

\author{
Independent Researcher \\ Received: August 11, 2017 \\ doi:10.5539/cco.v6n2p12
}

\author{
Accepted: September 3, $2017 \quad$ Online Published: September 12, 2017 \\ URL: https://doi.org/10.5539/cco.v6n2p12
}

\begin{abstract}
The objective of the paper is to elucidate how interconnected biological systems can be better mapped and understood using the rapidly growing area of Big Data. We can harness network efficiencies by analyzing diverse medical data and probe how we can effectively lower the economic cost of finding cures for rare diseases. Most rare diseases are due to genetic abnormalities, many forms of cancers develop due to genetic mutations. Finding cures for rare diseases requires us to understand the biology and biological processes of the human body. In this paper, we explore what the historical shift of focus from pharmacology to biotechnology means for accelerating biomedical solutions. With biotechnology playing a leading role in the field of medical research, we explore how network efficiencies can be harnessed by strengthening the existing knowledge base. Studying rare or orphan diseases provides rich observable statistical data that can be leveraged for finding solutions. Network effects can be squeezed from working with diverse data sets that enables us to generate the highest quality medical knowledge with the fewest resources. This paper examines gene manipulation technologies like Clustered Regularly Interspaced Short Palindromic Repeats (CRISPR) that can prevent diseases of genetic variety. We further explore the role of the emerging field of Big Data in analyzing large quantities of medical data with the rapid growth of computing power and some of the network efficiencies gained from this endeavor.
\end{abstract}

Keywords: biotechnology, network effects, big data, CRISPR, gene, rare disease, mutation, cancer

\section{Introduction}

The term Big Data refers to a bunch of techniques used for analyzing oceans of data. The exponential advancement of computing power in recent decades has made the development and practical application of these techniques possible. The $3 \mathrm{Vs}$ it works with - large volumes of data, high velocity of data, and diverse variety of data (Erl et al., 2016) - will lead to interesting discoveries that were not possible before. In this paper, we will illustrate how the field of medical sciences can immensely benefit from these developments. Big Data involves working with data sets and correlating them to find interesting patterns and relationships. These techniques are used to efficiently process large quantities of data, analyze data in real time, compute data from diverse sources, and extract value from efficient data processing. We can observe the deployment of Big Data in the retail sector where better and more targeted advertisements and product or service promotions can be achieved by quickly correlating credit card data and online site visits.

\section{Modifying Biological Processes: The Jump to Biotech}

Modern medicine, as we see it today, began with the advent of pharmacology, which involves the study of drugs and their effects on the body. For a long time in modern medical history, drug development involved the trial and error method (Ray, 2017) of combining different molecules and understanding their effects on the body; this essentially involved understanding the chemistry of drugs to produce cures. The science of pharmacology relies on the combination of chemicals or molecules to find cures for ailments and diseases. Pharmaceutical companies use chemicals to make drugs. The bulk of the field concentrated primarily on combining different chemicals in drugs and testing them first on animals and finally on humans. Gradually, this process of trial and error used in pharmacology for drug development was augmented by a more sophisticated method which involved the modification of biological processes.

Biotechnology has seen significant growth since the 1980s, which marked the beginning of biotechnology revolution. As the evolutionary successor of pharmacology, it modifies biological processes and hence requires a more detailed and intrusive understanding of the human body. Biotechnology alters and manipulates the genetics of organisms to come up with cures and treatments for diseases. The subject requires one to have a deep 
understanding of the biological processes in a human body as well as microorganisms to be able to come up with products that can be used in the treatment of diseases.

The pace of innovation has reduced in recent years and revenue generation from selling pharmaceutical drugs has slowed down and is becoming a less important part of bottom line for traditional pharma companies. Their attention is shifting towards acquiring biotechnology companies that have seen massive growth in recent decades. Pharmaceutical giants such as Pfizer, Merck \& Co, and Abbott Laboratories have all acquired biotechnology companies over the years and transformed themselves into a new breed of multinationals called biopharmaceuticals. Some of the prominent biotechnology companies such as Amgen, AbbVie, and Gilead Sciences have gained significant market share and their profitability is growing. (Gatlin, 2017). Pharmaceutical companies are acquiring growth and expertise in the field of biotechnology through mergers and acquisitions, as seen in the recent takeover of Medivation by Pfizer and Actelion by Johnson and Johnson. Growth in pharmacology has slowed down and more significant advancements are coming from the field of biotechnology. In their paper titled "Pharmaceutical Companies and the Transition to Biotechnology: A Study in Strategic Innovation" by Louis Galambos and Jeffrey L. Sturchio highlight the increased importance of biotech companies in the pharmaceutical industry. The paper gives us useful insights into how pharmaceutical manufacturers are establishing and augmenting capabilities in the field of biotechnology. (1998).

In an age where it's becoming easier and easier to generate medical data, the need of the hour is to develop sophisticated tools for processing that data in an efficient manner. Rapidly increasing processing power and development of sophisticated algorithms extend the scope for computation of large and varied data sets. So how do you link vast quantities of data? How do you link this diverse knowledge base into the mix? How do you bring massive amounts of data sitting in cold storage into use? Big Data essentially finds a solution to this problem. Many analysis techniques are available in the toolbox for large-scale application. This includes Euclid's algorithms, Euler's graph theory, chaos theory, Gaussian copula, Fibonacci's numbers, Boolean logic, and Bernoulli's law of large numbers, to mention a few. There is immense potential for endless expansion of Big Data's tool kit. (Steiner, 2012).

\section{Exploiting Network Effects}

Creativity, an elusive enigma, requires both depth of knowledge and diversity of knowledge to shine. The interaction between different disciplines, pieces of ideas, and fragments of knowledge is responsible for this paradox. If we dare to draw bold parallels, network effects work a bit like human creativity, a combustible cocktail that spews out new ideas. The $21^{\text {st }}$ century tool that makes creativity possible in an age of machines is scientifically termed Big Data. Big Data gains from network efficiencies. A fine example of network effects comes from cell phone users. The greater the number of people who use cell phones, the more valuable your cell phone is to you since you can talk to a lot more people - i.e. the more valuable the cellular network is to each user. (Anderson, 2009). The addition of new users increases the value of the network exponentially. Applying the same logic in information systems, the addition of new piece of information makes the information system as a whole more valuable. This phenomenon is termed network effects. Similar to how math makes understanding the universe easier by reducing it to a bunch of equations, Big Data techniques can spark our understanding of the human body.

Biotechnology gains tremendously from network effects. The more the body of knowledge is expanded around the area of biological systems and processes, the greater the value of each sphere of knowledge is to others. Each body of knowledge gains its value from the development of other bodies and the growth of new bodies of knowledge. Our understanding of human biochemistry can improve our understanding of physiology. A better understanding of Microbiology, the study of microorganisms, enables us to make advances in Immunology, the study of our immune system. The value of the network is dependent on its constituting members, and these network efficiencies can be harvested to accelerate biomedical innovations.

Another important factor that will improve the network effects is the diversity of data. The more diverse the data sets we can work with are, the more apparent the benefits that can be gained through Big Data. The diversity of data strengthens the knowledge base which can be leveraged for finding strong/weak or constant/transient relationships. Let's take the example of surveillance. To find and catch a criminal, it's not enough to merely collect and store data; one needs to properly index it and correlate it with other sets of data like IP addresses, phone calls, addresses, credit card data, etc. for reliable and actionable results. Many papers are published in the area such as "Strategic Alliances in the Global Biotechnology - Network Approach" by Per Håkansson and others that explore the need for strategic alliances among biotechnology companies to reap efficiencies. (Håkansson et al.,1993).

In contrast to the science of pharmacology which merely studies the effect of drugs on biological systems, the field 
of biotechnology draws on the vast libraries of knowledge on biological systems to develop solutions and benefits immensely from the expansion of its knowledge base. The value of an information network is dependent on how effectively we can link one set of data with other sets of data. Building a solid foundation of knowledge is essential to reap the benefits of network efficiencies. Network effects expand knowledge by tying pieces together to make the whole more than the sum of its parts. As more knowledge is built around different subjects, easier it becomes to find solutions.

Network effects can be reaped by:

- Researching a wider range of biological processes.

- Researching into areas that are deemed economically infeasible such as rare diseases, but may have incremental benefit and escalate the field of study in the long term.

- Researching areas that have not been explored previously such that low hanging fruits can be harvested.

- Conducting long-term medical studies that can give insights into the working of the human body around the effects of different medicines through sophisticated computation techniques.

\section{Status Quo: Rare Diseases}

About 5\% of the world's population is currently living with some form of rare disease. A disease is described rare if it afflicts fewer than 5 in 10000 people in Europe or fewer than 200,000 people in the US. (Aronson, 2006). For the US population of 320 million people, 30 million are affected by some form of orphan disease, which is equivalent to 1 in 10 in the population. An estimate of over 7000 such diseases and conditions are recognized in the world with extremely high percentage (95\%) of them having no FDA approved drug treatment. (Bavisetty et al., 2013). For people with orphan diseases, the mean average duration of time for symptoms to appear to enable an accurate determination is approximately 4.8 years. (Engel et al., 2013). Likewise, a rare disease may be critical or persistently crippling. (DG SANCO, n.d.). People with a orphan disease may be subjected to lower quality of life and suffer disabilities. Misdiagnosis and non-diagnosis impede the quality of life for such patients. (Lim et al., 2010).

\section{Advancing Genetics}

Around $80 \%$ of these orphan diseases have genetic roots. (Bavisetty et al., 2013). Genetic mutations such as Aplasias which result in deficient development or absence of an organ or tissue (Aplasia - Symptoms, Causes, Treatment, n.d.) and Ichthyosis (commonly called cracked skin) which looks like the scales of a fish are some such non-cancerous genetic diseases. (Ichthyosis, n.d.). Most rare diseases are due to genetic mutation. For instance, cystic fibrosis which affects the lungs occurs due to the mutation of CFTR gene. (GARD, n.d.). The same holds true for medical conditions such as dwarfism and color blindness. Many sarcomas or cancers arise from cell transformation and mutations. A women's increased risk of developing hereditary ovarian or breast cancer later on in life is due to genetic mutations of BRCA1 and BRCA2 genes. (NCI, n.d.).

Medical advancements have been few and far between in this area. Only 400 products have been approved to be used as a treatment in the US and only 70 in the EU. ("European Regulation on Orphan Medicinal Products", 2011 ; Braun et al., 2010). On average, clinical trials for rare diseases on human participants cost thousands of percent more per patient than other commonly known diseases. Too few patients, geographically dispersed patient population, insufficient information around indicators (biomarkers), and a composite of limited endpoint data sets are a few factors that has constrained the clinical proficiency for rare diseases. (WHO, 2013). There are many reasons for this underperformance in the study of rare diseases, one of which is that data is hoarded and not shared with the wider community.

The cost of conducting research on rare diseases and their cure may be extremely high, but the findings of such an endeavor can unlock a treasure trove of data points that highlight a significant deviation from what is considered normal. This builds a body of empirical relationships between diseases or disorders and genetic structures. Genetic research of this kind will surely lead to the unprecedented expansion of knowledge base of human biology which ultimately contributes to the long-term acceleration of biomedical solutions. A deep understanding of genes essentially enhances our understanding of the human body which, in turn, makes developing cures a more informed process. CRISPR (Clustered Regularly Interspaced Short Palindromic Repeats) is a popular method of manipulating the genes through genetic surgery. (Galeon, 2017). It acts as a preventive care for people who are at a greater risk of contracting a particular disease.

\section{Collaborative Research}

Many of the recent studies published in the New England Journal of Medicine emphasize the importance of 
sharing data and pushing for a more collaborative approach to research. The paper titled "Advantage of Truly Open-access Data Sharing Model" talks about sharing clinical trials in oncology research with multiple institutions. Cancer treatment trials funded by the National Cancer Institute (NCI) called National Clinical Trials Network (NCTN) has around 19000 cancer patients participating in the program with further 70000 patients who are funded by the pharmaceutical industry each year. (Bertagnolli et al., 2017).

In their paper titled "Data Sharing - Is Juice Worth the Squeeze?" Brian L. Strom and others discusses the benefits of sharing patient level data from clinical trials. (Storm et al., 2016). "Toward a Shared Vision for Cancer Genomic Data" by Robert L. Grossman and others talks about sharing raw genomic data including diagnostic, histologic, and clinical outcome data through Genomic Data Commons (GDC), a data repository developed by a consortium that includes National Cancer Institute (NCI), University of Chicago, Ontario Institute of Cancer Research, and Leidos Medical Research. (Grossman et al., 2016).

The establishment of various programmes and networks in recent years has also helped medical practitioners to make inroads in the diagnosis and care of rare diseases. (Aymé, 2012). The International Rare Diseases Research Consortium (IRDiRC) was spearheaded in 2011 by the European Commission along with U.S. National Institutes of Health with the sole aim of fostering international collaboration in rare diseases research. (WHO, 2013). With the advent of sophisticated healthcare database, information can now be accessed by doctors and patients alike.

Long-term studies that fully incorporate the effects of medication are scarce in the field of medical sciences. Long-term studies can provide rich data sets and a more comprehensive understanding of long-term biological processes. Such studies can plug knowledge gaps with vital data, thereby offering the medical field a colossal prospect of advancement.

Finding cures for rare diseases may not be a utopian affair like many might think, but a pursuit that adds to our overall understanding of the human body. Contrasting data points from studying rare diseases can help build statistical evidence of correlation and causality. Distinct sets of data can give different vantage points on how we view and interpret results. Learning about rare diseases will bring diversity to our knowledge base which will enable us to pluck the low hanging fruits of discovery.

\section{Diagnostics}

It is estimated that $75 \%$ of rare diseases affect children and have an remarkably high mortality rate of $30 \%$ for children below 5 years of age. (Lim et al., 2010). About $40 \%$ of orphan disease patients are misdiagnosed no less than once. (Engel et al., 2013). Detection of these medical conditions can be an exceedingly burdensome procedure as an orphan disease is misdiagnosed 3 times on average before the genuine diagnosis. Moreover, it takes an average of 8 consultations with doctors and more than 7 years before genuine diagnosis of a orphan disease is established. The longer it takes to precisely diagnose a rare disease, the more doctor visits the patient needs to make. (Shire, 2013). This uncertain journey of diagnosis and care can inflate medical costs to prohibitive levels. (Engel et al., 2013).

Big Data which has played a major role in the area of analytics can extend its usefulness to biotechnology research as well. The new field of study centered on DNA sequencing was a result of the surge in computing power. If we look at the history of diagnostics since the late 1800 s, we can see that X-rays merely provided 2-dimensional images of the internal parts and organs of the human body. Later, CT scans came into widespread use which can produce images of 3-dimensional cross-section of few millimeters by laying a bunch of traditional 2-dimensional $\mathrm{X}$-ray images one on top of the other using computers. Extending this computational progression to the extreme means there will come a time when every single cell in your body can be scanned and analyzed for abnormalities and cancerous mutations.

\section{Interconnected Biological Systems}

As computers can take on the herculean task of sifting through voluminous data, we can study and understand the interconnectedness of biological systems. Now that vast amounts of data need not go into cold storage, everything can be cached to call on vast databases. The massive amounts of data we are generating around human biology require strong analysis techniques to find statistically significant relationships to derive value. By building systems to overlay different data points, usable information can be extracted to produce clear diagnosis and recommend treatments.

Network efficiencies can be gained from a systems approach to understanding biology. In their paper titled "The Impact of Systems Approaches on Biological Problems in Drug Discovery" by Leroy Hood and Roger M. Perlmutter states that "the application of systems approaches to drug discovery promises to have a profound impact on medical practice, permitting a comprehensive evaluation of underlying predisposition to disease, 
disease diagnosis and disease progression". (2004, p. 1215).

Network effects construct bridges between vertical knowledge silos. Understanding a disease's impact on the body can make the development of cures for the same easier. Developments in diagnostic care can make headways in the field of preventive care. There are spillover effects to detection and prevention, like advancements in diagnostic tests can improve screening tests; developments in the diagnostics for cancer can improve its rate of detection. Developing cure for a particular disease can make developing cures for similar ailments more straightforward. By mapping the DNA of people, medical practitioners can give them early warnings regarding the increased risk of developing a particular disease as well as make way for more targeted cures.

\section{Going Forward}

Medical sciences are the next frontier where technology will have its crucial influence. Biotech is ripe for disruption from Silicon Valley technology companies. Today we are seeing a lot of wearable devices receiving widespread adoption in the market. Medical sciences are set to experience the next wave of innovation where the process of finding solutions involves large-scale management of data. IBM's supercomputer Watson that won Jeopardy in 2011 is basically a high tech data retrieval system where data is indexed to be accessed quickly. (Kaplan, 2015). Integration of information databases will be the name of the game and data analytics will have a more pronounced role in the coming years. Today, Watson is repurposed for tackling real world problems such as medical diagnostics, genomics, and drug discovery. (IBM Watson Health, n.d.).

A recent article in the Harvard Business Review titled "An Online Medical Database is Reducing Diagnostic Errors" talks about how proof based medical content accessible to caregivers can enhance diagnostic reliability. (Weintraub et al., 2017). A recent article in the New England Journal of Medicine titled "Predicting the Future Big Data, Machine Learning, and Clinical Medicine" talks about the importance of machine learning to enhance predictability and prognosis, which can help take over the work of radiologists and anatomical pathologists. This, in a nutshell, will improve diagnostic accuracy beyond human threshold. (Obermeyer, \& Emanuel, 2016).

Study of genetics, a field that will see a vast expansion is the future of medical sciences, will accelerate innovations in biotechnology. Some of the frontiers of medical advancement like gene therapy and customized medicine which are at the cutting edge of medical sciences, although accessible to few, will have spillover benefits of building a knowledge base that will propel other areas of medical advancement. An assortment of knowledge is essential to have network efficiencies working in our favor by strengthening knowledge around anatomy, physiology, biochemistry, pathology, microbiology, pharmacology, pediatrics, etc.

\section{Conclusion}

The informational efficiencies harvested by studying rare diseases grossly outweighs the economic costs of finding cures. The Spillover benefits from researching into rare diseases can squeeze costs in the long term of wider medical research. Building a solid foundation of knowledge is essential to escalate the field of medical study. Linear growth in constructing a body of knowledge corresponds to the geometric advances in developing cures. Exploring the DNA will bring a windfall in accelerating knowledge generation, adding new branches of knowledge, improving diagnostics and preventive care, and precipitating exponential innovation in the long term for medical sciences. By building a body of literature on the subject which extends deep into the sciences, we can bring forth incremental discoveries and build an empirical foundation for future inventions.

\section{References}

Anderson, C. (2009). ECON 000: How a Century-old Joke Became the Law of Digital Economics. In Free: The Future of a Radical Price (pp. 137-146). London: Random House Business Books.

Aplasia-Symptoms, Causes, Treatment. (n.d.). Retrieved June 21, 2017, from http://symptomscausestreatment.com/aplasia-symptoms-causes-treatment.html

Aronson, J. K. (2006, March). Rare Diseases and Orphan Drugs. British Journal of Clinical Pharmacology. https://doi.org/10.1111/j.1365-2125.2006.02617.x

Aymé, S. (2012). State of the Art of Rare Disease Activities in Europe: A EUCERD Perspective. Orphanet Journal of Rare Diseases, 7(Suppl 2). https://doi.org/10.1186/1750-1172-7-S2-A1

Bavisetty, S., Grody, W. W., \& Yazdani, S. (2013). Emergence of Pediatric Rare Diseases. Rare Diseases, 1(1). https://doi.org/10.4161/rdis.23579

Bertagnolli, M. M., Sartor, O., Chabner, B. A., Rothenberg, M. L., Khozin, S., Hugh-Jones, C., Reese, D. M., \& Murphy, M. J. (2017). Advantages of a Truly Open-Access Data-Sharing Model. New England Journal of Medicine, 376(12), 1178-1181. https://doi.org/10.1056/NEJMsb1702054 
Braun, M. M., Farag-El-Massah, S., Xu, K., \& Coté, T. R. (2010). Emergence of Orphan Drugs in the United States: A Quantitative Assessment of the First 25 Years. Nature Review Drug Discovery, 9(7), 519-522. https://doi.org/10.1038/nrd3160

BRCA1 and BRCA2: Cancer Risk and Genetic Testing. (n.d.). Retrieved from https://www.cancer.gov/about-cancer/causes-prevention/genetics/brca-fact-sheet

California Healthcare Institute. (n.d.). Combating Rare Diseases in the 21st Century. California. Retrieved from https://califesciences.org/wp-content/uploads/2015/05/Rare-Disease-Brochure-1-Page-Version1.pdf

Cystic Fibrosis. (n.d.). Retrieved from https://futurism.com/berkeley-biologist-crispr-gene-editing-will-cure-genetic-disease/

Engel, P. A., Bagal, S., Broback, M., \& Boice, N. (2013). Physician and Patient Perceptions Regarding Physician Training in Rare Diseases: The Need for Stronger Educational Initiatives for Physicians. Journal of Rare Diseases, 1(2), 1-4. Retrieved from http://www.journalofraredisorders.com/pub/IssuePDFs/Engel.pdf

Erl, T., Khattak, W., \& Buhler, P. (2016). Big Data Fundamentals: Concepts, Drivers \& Techniques. NJ, USA: Prentice Hall Press.

European Commission: Health \& Consumer Protection Directorate-General. (n.d.). Useful Information on Rare Diseases from an EU Perspective. Retrieved from http://ec.europa.eu/health/ph_information/documents/ev20040705_rd05_en.pdf

European Regulation on Orphan Medicinal Products: 10 Years of Experience and Future Perspectives. (2011). Nature Review Drug Discovery, 10(5), 341-349. https://doi.org/10.1038/nrd3445

EURORDIS. (2009). The Voice of 12,000 Patients: Experiences and Expectations of Rare Disease Patients on Diagnosis and Care in Europe. Retrieved from http://www.eurordis.org/IMG/pdf/voice_12000_patients/EURORDISCARE_FULLBOOKr.pdf

Galambos, L., \& Sturchio, J. L. (1998). Pharmaceutical Firms and the Transition to Biotechnology: A Study in Strategic Innovation. Business History Review, 72(2), 250-278. https://doi.org/10.2307/3116278

Galeon, D. (2017, June 8). Berkeley Biologist: “CRISPR Gene Editing Will Cure Genetic Disease.” Futurism. Retrieved from https://futurism.com/berkeley-biologist-crispr-gene-editing-will-cure-genetic-disease/

Gatlin, A. (2017, February 23). AbbVie Stronger Bet than Gilead, Amgen-At Least until 2020: Leerink. Investor's Business Daily. Retrieved from http://www.investors.com/news/technology/abbvie-stronger-bet-than-gilead-amgen-at-least-until-2020-leeri $\mathrm{nk} /$

Grossman, R. L., Heath, A. P., Ferretti, V., Varmus, H. E., Lowy, D. R., Kibbe, W. A., \& Staudt, L. M. (2016). Toward a Shared Vision for Cancer Genomic Data. New England Journal of Medicine, 375(12), 1109-1112. https://doi.org/10.1056/NEJMp1607591

Hood, L., \& Perlmutter, R. M. (2004). The Impact of Systems Approaches on Biological Problems in Drug Discovery. Nature Biotechnology, 22(10), 1215-1217. https://doi.org/10.1038/nbt1004-1215

IBM Watson Health. (n.d.). Retrieved from https://www.ibm.com/watson/health/

Ichthyosis. (n.d.). Retrieved June 19, 2017, from http://www.aocd.org/?page=ichthyosis

Kalyan Ray. (2017, May 22). New Path for Drug Development. Deccan Herald. Retrieved from http://www.deccanherald.com/content/613033/path-drug-development.html

Kaplan, J. (2015). Humans Need Not Apply: A Guide to Wealth and Work in the Age of Artificial Intelligence. New Haven, CT: Yale University Press. Retrieved from https://books.google.co.in/books?id=yatJCgAAQBAJ

Kinch, M. S. (2014). The Rise (and Decline?) of Biotechnology. Drug Discovery Today, 19(11), 1686-1690. https://doi.org/10.1016/j.drudis.2014.04.006

Limb, L., Nutt, S., \& Sen, A. (2010). Experiences of Rare Diseases: An Insight from Patients and Families. Retrieved from http://www.raredisease.org.uk/media/1594/rduk-family-report.pdf

Obermeyer, Z., \& Emanuel, E. J. (2016). Predicting the Future-Big Data, Machine Learning, and Clinical Medicine. New England Journal of Medicine, 375(13), 1216-1219. https://doi.org/10.1056/NEJMp1606181

Per Håkansson, Hans Kjellberg, \& Anders Lundgren. (1993). Strategic Alliances in Global Biotechnology-A 
Network Approach. International Business Review, 2(1), 65-82. https://doi.org/10.1016/0969-5931(93)90005-H

Shire. (2013). Rare Disease Impact Report: Insights from Patients and the Medical Community. Retrieved from https://globalgenes.org/wp-content/uploads/2013/04/ShireReport-1.pdf

Steiner, C. (2012). Automate This: How Algorithms Took Over Our Markets, Our Jobs, and the World. New York: Penguin Publishing Group.

Strom, B. L., Buyse, M. E., Hughes, J., \& Knoppers, B. M. (2016). Data Sharing-Is the Juice Worth the Squeeze? New England Journal of Medicine, 375(17), 1608-1609. https://doi.org/10.1056/NEJMp1610336

Weintraub, R., Bonis, P., \& Valtis, Y. K. (2017, October). An Online Medical Database Is Reducing Diagnostic Errors. Harvard Business Review. Retrieved from https://hbr.org/2015/10/an-online-medical-database-is-reducing-doctor-error

World Health Organization. (2013). Priority Medicines for Europe and the World Update Report. Retrieved from http://www.who.int/medicines/areas/priority_medicines/en/

\section{Copyrights}

Copyright for this article is retained by the author(s), with first publication rights granted to the journal.

This is an open-access article distributed under the terms and conditions of the Creative Commons Attribution license (http://creativecommons.org/licenses/by/4.0/). 